\title{
The Utilisation of Marrow Concentrate in Canine
}

\section{Arthrosis-Case Report}

Busuioc Diana-Alexandra ${ }^{1}$, Șindilar Eusebiu-Viorel ${ }^{1}$, Horhogea Cristina ${ }^{1}$, Lăbușcă (Simion) Luminița ${ }^{2}$ and Drugociu Dan ${ }^{1}$

1. University of Agricultural Sciences and Veterinary Medicine, Iași, Faculty of Veterinary Medicine, 3 Mihail Sadoveanu Alley, Iași 700490, Romania

2. Orthopedics and Traumatology Clinic County Emergency Hospital 1, Independentei Boulevard, 70111, Iasi, Romania

\begin{abstract}
The use of bone marrow concentrate in the treatment of degenerative joint disese (DJD) in dogs represents an innovative approach, minim invasive, that already has been used with increasing success in the veterinary field. The technique, adapted from human medicine for the quadruped patients is based on the principle of the autologous transplant of mesenchymal stem cells that are included in the bone marrow concentrate. The intervention involves a short anesthesia of the animal for harvesting the marrow. The processing of the marrow is realised in strict aseptic conditions in a laboratory and the inoculation is done in the same day. The results are clinically visible after 2-4 weeks. For an enhanced effect, a part of the concentrate is grown on a flask in specific conditions in order to expand autologous bone marrow stem cells and is inoculated after a sufficient number of cells are achieved, usually 3-4 weeks.
\end{abstract}

Key words: Arthrosis, marrow concentrate, mesenchymal stem cells.

\section{Introduction}

One of the definitions of the regenerative medicine given by Greenwood et al. [1] is an emerging interdisciplinary field of research and clinical application focused on the repair, replacement or regeneration of cells, tissues or organs to restore impaired function resulting from any cause, including congenital defects, disease, trauma and aging. It uses a combination of several technological approaches that move it beyond traditional transplantation and replacement therapies. These approaches may include, but are not limited to, the use of soluble molecules, gene therapy, stem cell transplantation, tissue engieneering and the reprogramming of cell and tissue types.

Mesenchymal stem cells (MSCs) have been successfully isolated, from human and animal patients, from a large variety of tissue: bone marrow [2],

Corresponding author: Busuioc Diana-Alexandra, DVM, MRCVS, Ph.D. student, research fields: veterinary medicine and area of research canine arthrosis. placenta, oumbilical cord, blood, adipose tissue [3]. MSCs are multi-potent and can differentiate into a large range of cells, from those of mesodermal lineage: osteoblasts, chondrocytes and adipocytes, to neurocytes and cardiomyocytes [4].

Bone marrow mesenchymal stem cells are thought to be useful for reconstructing injured tissue such as bone, cartilage, or cardiac muscle [5]. This procedure is easy to perform clinically because the autologous bone marrow mesenchymal cells are easy to obtain and can be culture expanded without losing their capacity for differentiation $[5,6]$.

Here, we tried to use autologous bone marrow mesenchymal stem cells to repair a large cartilage defect in a hip joint of a canine patient with osteoartritis (OA).

\section{Methods and Materials}

The studied patient was a neutered male Husky, 10 years old who was previously diagnosed with degenerative joint disease (also known as osteoarthritis, 
OA) of the right hip joint. After different treatment options were discussed with the owners, the inoculation of autologous MSCs was agreed upon. However, if the inoculation did not result in the resolution of lamenes, or the clinical signs would reappear after a few months, the procedure can be repeated by culturing the MSCs harvested and stored at the time of the first collection.

The procedure had 3 steps: the harvest of the bone marrow, the separation of the MSCs from the marrow harvested and the inoculation of the separated MSCs in patient's hip joint.

To ensure that the patient can undergo anesthesia, he has undergone a clinical examination and blood had been collected for haematological and biochemistry analysis.

\subsection{Harvesting the Bone Marrow}

The patient had been fasted 12 hours prior to the procedure. A peripheral intravenous catheter was placed and an isotonic electrolyte solution $(0.9 \% \mathrm{NaCl})$ was administered at a maintainance rate. $\mathrm{He}$ was pre-medicated with buthorphanol $(0.1 \mathrm{mg} / \mathrm{kg})$ and acepromazine $(0.1 \mathrm{mg} / \mathrm{kg})$ by intramuscular injection. The induction was made with propofol $(4 \mathrm{mg} / \mathrm{kg})$. An endotracheal tube was placed and the aneasthesia was maintained with isoflurane.

Common sites for bone marrow aspiration in dogs are: the dorsal iliac crest, the trochanteric fossa medial to greater trochanter of the femur and the lateral aspect of the femoral head. For this procedure, the bone marrow was aspirated from the trochanteric fossa medial to the greater trochanter of the femur. The area was clipped and surgically prepared with a sterile scrub. All the procedure was executed in strict aseptic conditions. A Jamshidi type needle was used to collect the bone marrow as in Fig. 1. The needle was inserted through the surgically prepared skin, through the muscles, into the cortex and advanced for an additional $1 \mathrm{~cm}$. Ten $\mathrm{mL}$ of bone marrow was aspirated in a heparinised syringe.

\subsection{The Separation of the MSCs from the Bone Marrow}

Under a laminar flow hood, the blood was divided into 4 sterile centrifuge tubes and an equal quantity of separation solution was added in each tube (Histopaque-1119, Sigma Aldrich). The tubes were centrifuged 30 minutes at $400 \mathrm{~g}$. After centrifugation, the upper layer was aspirated with a Pasteur pipette at a distance of $0.5 \mathrm{~cm}$ from the opaque interface containing the mononuclear cells and removed. The opaque interface was transferred to a clean centrifuge tube and was washed by addition of several $\mathrm{mL}$ of DMEM culture medium (Dulbecco's Modified Eagle Medium, Sigma Aldrich), then the tubes were centrifuged again for 10 minutes at $250 \mathrm{~g}$. The cell pellet was resuspended with cell culture medium, mixed, then centrifuged for the 3rd time for $10 \mathrm{~min}$. The mononuclear cell layer obtained was resuspended in a small amount of culture medium. Of the quantity obtained, a small part is intended for cultivation under laboratory conditions in a cell culture flask and stored in case that a further inoculation is needed. The viable cells were counted using an automated cell counter, the result obtained was $5.2 \times 10^{6}$ viable cells $/ \mathrm{mL}$.

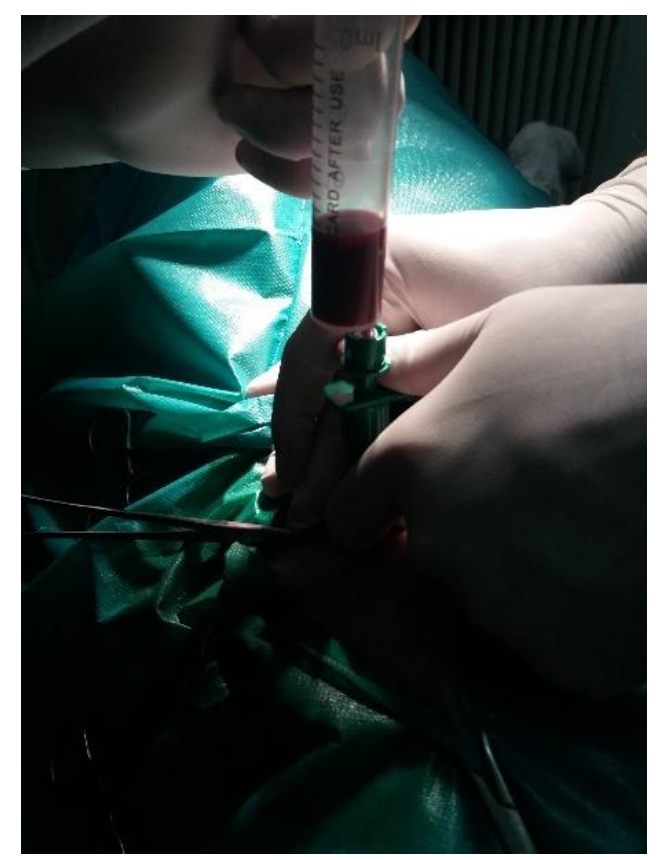

Fig. 1 Aspiration of bone marrow from the trochanteric fossa of a canine patient using a Jamshidi needle. 


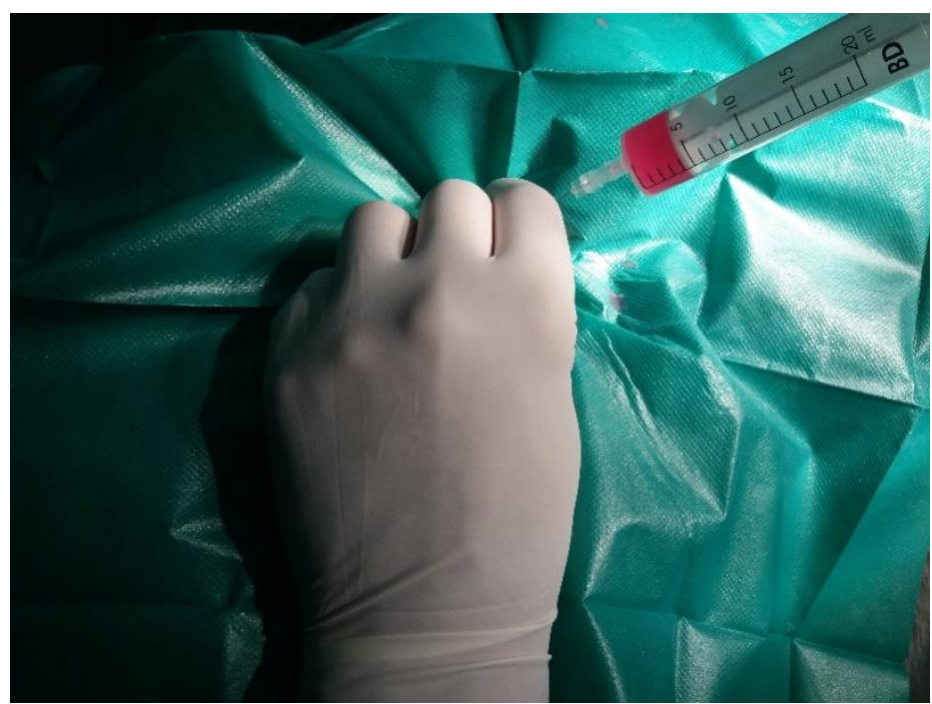

Fig. 2 The inoculation of the separated MSCs in the canine hip joint affected by OA.

\subsection{The Inoculation of the Separated MSCs in the Patient's Hip Joint}

The hip area was clipped and prepared as for surgery. The procedure was performed aseptically. The patient was placed in lateral recumbency, with the affected hip joint up, the needle being introduced in a cranio-caudal, latero-medial manner in the cranial part of the joint, as in Fig. 2.

The analgesia provided was carprofen $(2 \mathrm{mg} / \mathrm{kg})$ by mouth, every 12 hours, up to three days post procedure.

\section{Results and Discussion}

Initially, the lameness worsened for the 36 hours following the inoculation, but this was treated with success with NSAIDs.

For the first two weeks, our patient's exercise was restricted to 10-minute walks three times a day. At the two-week examination, the lameness had resolved completely and all analgesic treatment was stopped. There was no joint effusion palpable in the hip joint and no pain on manipulation of the joint.

Exercise was increased gradually over the next weeks, similar findings being reported at 4 weeks and 12 weeks check-ups. No stiffness or lameness of the right hindlimb were reported by the owners after 6 months.
The therapy with MSCs is not a cure for DJD, but the goal is to restore the affected joint to as near to pain-free normality as possible. There are different procedures that can be applied for harvesting and separating the MSCs from the bone marrow. Cases suitable for stem cell treatment can vary from younger to older patients, suffering from DJD or tendinopathies that have failed to be controlled by conventional treatment, or surgery is not an option. Mesenchymal stem cells can be stored and repeat therapies can be generated if needed. Many dogs are receiving one injection, while other need top-ups twelve to 18 months later.

\section{Conclusions}

There is plenty of evidence that regenerative medicine has beneficial effects, both in humans and in animals. From all the animal species, stem cell therapy is frequently applied in horses and dogs. Although the results are rewarding for both veterinary surgeons and for the owners, the scientific community is still awaiting a placebo-controlled blinded trial, considered the gold standard, the benefits of the stem cell therapy. Different practices in the USA and UK are currently using stem cell therapy as a routine for the patients suffering from DJD, but as far as we are aware, our patient was within the first cases treated in Romania. 


\section{References}

[1] Greenwood, H. L., Thorsteinsdottir, H., Perry, G., Renihan, J., Singer, P. A., and Daar, A. S. 2006. "Regenerative Medicine: New Opportunities for Developing Countries.” Int. J. Biotechnol. 8 (1-2): 60-77.

[2] Pittenger, M. F., Mackay, A. M., Beck, S. C., Jaiswal, R. K., Douglas, R., Mosca, J. D., et al. 1999. "Multilineage Potential of Adult Human Esenchymal Stem Cells." Science 284: 143-7.

[3] Sanchez-Ramos, J. R. 2002. "Neural Cells Derived from Adult Bone Marrow and Umbilical Cord Blood." $J$ Neurosci Res 69: 880-93.
[4] Hiroshi, T., Dongwei, Z., Ichiro, Y., Yusuji, H., Masaki, M., and Toshiro, A. 2012. "Comparison of Bone Marrow and Adipose Tissue-Derived Autologous Culture Expanded Bone Marrow Mesenchymal Cell Transplantation for Repair of Cartilage Defects in Osteoarthritic Knees." J of OsteoArthritis Research Society Int. 10: 199-206.

[5] Wakitani, S., Imoto, K., Yamamoto, T., Saito, M., Murata, N., and Yoneda. 2002. "Human Canine Mesenchymal Stem Cells." BMC Veterinary Research 8: 150.

[6] Caplan, A., Elyaderani, M., Mochizuki, Y., Wakitani, S., and Goldberg, V. M. 1997. "Principles of Cartilage Repair and Regeneration." Clin Orthop. 342: 254-69. 